\title{
AAVD Regimen
}

National Cancer Institute

\section{Source}

National Cancer Institute. AAVD Regimen. NCI Thesaurus. Code C160563.

A chemotherapy regimen consisting of brentuximab vedotin, doxorubicin, vinblastine and dacarbazine that can be used in the treatment of Hodgkin lymphoma (HL). 